\title{
Adaptive Finite-Time Command Filtered Fault-Tolerant Control for Uncertain Spacecraft with Prescribed Performance
}

\author{
Zhongtian Chen $(\mathbb{D}$, Qiang Chen $(\mathbb{D}$, Xiongxiong He, and Mingxuan Sun \\ Data-Driven Intelligent Systems Laboratory, College of Information Engineering, Zhejiang University of Technology, \\ Hangzhou 310023, China \\ Correspondence should be addressed to Qiang Chen; sdnjchq@zjut.edu.cn
}

Received 17 August 2018; Accepted 23 October 2018; Published 6 November 2018

Guest Editor: Junpei Zhong

Copyright (c) 2018 Zhongtian Chen et al. This is an open access article distributed under the Creative Commons Attribution License, which permits unrestricted use, distribution, and reproduction in any medium, provided the original work is properly cited.

In this paper, an adaptive finite-time fault-tolerant control scheme is proposed for the attitude stabilization of rigid spacecrafts. A first-order command filter is presented at the second step of the backstepping design to approximate the derivative of the virtual control, such that the singularity problem caused by the differentiation of the virtual control is avoided. Then, an adaptive fuzzy finite-time backstepping controller is developed to achieve the finite-time attitude stabilization subject to inertia uncertainty, external disturbance, actuator saturation, and faults. Through using an error transformation, the prescribed performance boundary is incorporated into the controller design to guarantee the prescribed performance of the system output. Numerical simulations demonstrate the effectiveness of the proposed scheme.

\section{Introduction}

Due to the significant role in guaranteeing the success of any spacecraft related mission, the attitude control of the spacecraft has obtained much attention, and numerous control schemes are proposed, such as adaptive control, sliding mode control, backstepping control, $H_{\infty}$ control, finite-time control, and so on [1-8]. Considering the specificity of the spacecraft's working environment, the hardware of the spacecraft is unlikely repairable, and the faults or failures cannot be fixed with replacement parts after the spacecraft is launched. The existence of faults or failures can potentially cause all kinds of safety, economic, and environment problems, and they should be considered in the attitude control design.

Different from the conventional control system without considering the possibility of fault occurrence, the faulttolerant control (FTC) can guarantee desirable performance properties even the actuators are not healthy. In general, the existing FTC technique can be roughly classified into two categories: active FTC and passive FTC. The active FTC relies on the fault detection and diagnosis (FDD) algorithm to provide the real-time information of the system status and then reconfigure the controller to achieve the control objective. For instance, an active fault-tolerant control was proposed for the flexible spacecraft in [9], such that the attitude stabilization was achieved. Unlike active FTC, by using a single fixed controller, passive FTC dose not require any online fault information and reconfiguration mechanism. Therefore, the passive FTC is more succinct, easy to compute and suitable to the actual application. Most of FTC controllers designed for the spacecraft attitude control are passive [1012]. In [10], an adaptive fuzzy fault-tolerant controller was proposed to achieve the spacecraft attitude tracking. A sliding mode control based attitude fault-tolerant controller was presented in [11], and the attitude was stabilized for the satellite with solar flaps. For those spacecrafts with redundancy actuators, the common method is using the actuator distribution matrix. In [12], a fault-tolerant control method based on distribution matrix was presented for the spacecraft attitude tracking, such that the finite-time convergence of the tracking error was achieved.

Actuator saturation is another issue worthy of study, the actual actuators in the spacecraft have the nominal limit of the output, and saturated output definitely effaces the system performance. Although there exist works concerning the attitude control design with actuator saturation [13-15], the possibility of the emerging of actuator failure at the same time is ignored. When the actuator fault happens, in order 
to maintain the performance of the system, the need for large control torque leads to severe actuator saturation. Until now, numerous works have considered both issues and designed controllers for the spacecraft attitude control [16-18].

Convergence speed is always significant in practically spacecraft system, and the finite-time control is able to provide faster convergence performance and higher tracking precision. Most of the finite-time control methods applied to the spacecraft have two kinds: homogeneous method $[19,20]$ and Lyapunov method [21-24]. In [19], a local continuous finite-time control scheme was proposed for the spacecraft system with an unknown inertia matrix and the homogeneous method based controller achieved attitude stabilization within a finite time. Homogeneous system theory was used to design a finite-time controller in [20], and the spacecraft attitude was stabilized within a finite time even with actuator saturation. In $[5,21]$, two adaptive terminal sliding mode controllers were proposed based on the Lyapunov method, such that the spacecraft attitude and angular velocity could converge to a small region of the origin within a finite time, respectively. Furthermore, the recent works [12, 16, 22] have achieved the finite-time spacecraft attitude stabilization or tracking in the presence of the actuator saturation and faults.

The adaptive backstepping approach $[25,26]$, as a recursive Lyapunov-based scheme, has emerged as a powerful method to construct controllers for nonlinear systems since early 1990s. There are several works using the backstepping method to design controllers of the spacecraft $[3,27]$; however, the closed-loop stability is achieved when time goes to infinity. When designing a finite-time backstepping controller, the differentiation of the virtual controller in recursive steps may lead to the singularity problem. Recently, the finitetime command filtered backstepping approach was proposed in $[28,29]$, where the first-order Levant differentiator was used to approximate the derivative of the virtual controller, such that the finite-time convergence can be achieved when the system model was known or partially known.

All of the aforementioned works mainly focus on the steady-state behavior but ignore the transient performance such as convergence rate and overshot. To achieve the specific goals of spacecraft missions which need a specific rotating speed or a limitation of angular velocity, prescribed transient performance of the system output is very important. The widely used techniques to improve transient performance mainly include barrier Lyapunov function (BLF) [30-33], funnel control [34-36], prescribed performance control (PPC) [37-42], and so on. In order to guarantee the prescribed performance imposed on the transient and steadystate output error, the prescribed performance control (PPC) method was proposed by Bechlioulis and Rovithakis for uncertain nonlinear systems [37]. For the spacecraft attitude system with input saturation, a PPC based adaptive faulttolerant control was presented in [43], such that the output of the system was constrained by the prescribed performance. Nevertheless, the designed controller in [43] could only guarantee the asymptotic uniform ultimate boundedness of the spacecraft system as the time goes to infinity.

Motivated by the aforementioned discussions, the fuzzy finite-time attitude stabilization problem for spacecraft systems under the actuator saturation and faults is studied in this paper, and a fuzzy finite-time fault-tolerant controller is proposed to achieve prescribed transient and steadystate performance of the system output. However, it is a challenging work to design a controller to guarantee the prescribed transient performance when considering the input constraints including actuator saturation and faults. On the one hand, when the initial state is far away from the equilibrium state, the required control input is usually set relatively large to guarantee the fast transient response. But on the other hand, due to the effect of the input saturation and actuator fault, it is a hard work to keep the satisfactory transient response as usual. The main contributions of this paper are listed as follows.

(1) A first-order command filter is presented at the second step of the backstepping design to approximate the derivative of the virtual control, such that the singularity problem caused by the differentiation of the virtual control is avoided.

(2) An adaptive fuzzy finite-time backstepping controller is developed to achieve the finite-time attitude stabilization subject to inertia uncertainty, external disturbance, actuator saturation, and faults.

(3) Through using an error transformation, the prescribed performance boundary is incorporated into the controller design to guarantee the prescribed performance of the system output.

The rest of this paper is organized as follows. Section 2 states the formulation of the spacecraft attitude stabilization problem. In Section 3, some preliminary knowledge and lemmas are given. In Section 4, the fuzzy finite-time faulttolerant control scheme is proposed and followed by stability analysis. Simulation results are provided in Section 5, and the conclusion is summarized in Section 6.

\section{Problem Formulation}

Considering the attitude stabilization problem for a rigid spacecraft, the modified Rodrigues parameter (MRP) based spacecraft system is described as [44]

$$
\begin{aligned}
\dot{\sigma} & =\frac{1}{4}\left[\left(1-\sigma^{T} \sigma\right) I_{3}+2 \sigma^{\times}+2 \sigma \sigma^{T}\right] \omega=G(\sigma) \omega \\
J \dot{\omega} & =-\omega^{\times} J \omega+u+d
\end{aligned}
$$

where $\sigma=\left[\sigma_{1}, \sigma_{2}, \sigma_{3}\right]^{T}$ is the spacecraft attitude in body frame with respect to the inertial frame presented by MPRs, $I_{3}$ is the identity matrix, and the operational symbol $a^{\times}$denotes the following skew-symmetric matrix for any vector $a=$ $\left[a_{1}, a_{2}, a_{3}\right]^{T}$

$$
a^{\times}=\left[\begin{array}{ccc}
0 & -a_{3} & a_{2} \\
a_{3} & 0 & -a_{1} \\
-a_{2} & a_{1} & 0
\end{array}\right]
$$

$\omega=\left[\omega_{1}, \omega_{2}, \omega_{3}\right]^{T}$ is the body frame angular velocity with respect to inertial frame. $J=J_{0}+\Delta J$ is the inertia matrix of the spacecraft, where $J_{0}$ denotes the nonsingular known nominal value of the inertia matrix and $\Delta J$ is the bounded uncertainty. 
$u$ and $d$ in (2) denote the control torque and the bounded external disturbance torque, respectively. Considering the input saturation and actuator faults in the rigid spacecraft, the actual control torque $u$ is further formulated as

$$
u=E \operatorname{sat}(v)
$$

where $E=\operatorname{diag}\left\{E_{1}, E_{2}, E_{3}\right\}$ with $0<E_{i} \leq 1 \quad(i=1,2,3)$ is the fault matrix and sat $(v)$ is the saturated control input satisfying $\operatorname{sat}(v)=\left[\operatorname{sat}\left(v_{1}\right), \operatorname{sat}\left(v_{2}\right), \operatorname{sat}\left(v_{3}\right)\right]$, where $v=\left[v_{1}, v_{2}, v_{3}\right]^{T}$ is the commanded control which needs to be designed later, $\operatorname{sat}\left(v_{i}\right)=\operatorname{sgn}\left(v_{i}\right) \times \min \left\{\left|v_{i}\right|, v_{M i}\right\}$ with $\operatorname{sgn}(\cdot)$ denoting the sign function and $v_{M i}$ being the $i$ th axis maximum torque.

The nonlinear saturation function $\operatorname{sat}\left(v_{i}\right)$ can be approximated by the following smooth function $g\left(v_{i}\right)$ :

$$
g\left(v_{i}\right)=v_{M i} \times \tanh \left(\frac{v_{i}}{v_{M i}}\right)=v_{M i} \frac{e^{v_{i} / v_{M i}}-e^{-v_{i} / v_{M i}}}{e^{v_{i} / v_{M i}}+e^{-v_{i} / v_{M i}}} .
$$

Then, $\operatorname{sat}(v)$ is rewritten as

$$
\text { sat }(v)=g(v)+d_{s}(v)
$$

where $g(v)=\left[g\left(v_{1}\right), g\left(v_{2}\right), g\left(v_{3}\right)\right]^{T}$ and $d_{s}(v)=\left[d_{s}\left(v_{1}\right)\right.$, $\left.d_{s}\left(v_{2}\right), d_{s}\left(v_{3}\right)\right]^{T}$ stands for the approximation error. It is noted that $d_{s}\left(v_{i}\right)$ is a bounded function and its bound satisfying $\left|d_{s}\left(v_{i}\right)\right|=\left|\operatorname{sat}\left(v_{i}\right)-g\left(v_{i}\right)\right| \leq v_{M i}(1-\tanh (1))$.

According to the mean value theorem, there exist constants $c_{i}\left(0<c_{i}<1\right), i=1,2,3$, such that the following inequality holds:

$$
g\left(v_{i}\right)=g\left(v_{i}^{\prime}\right)+g_{\mu}\left(v_{i}-v_{i}^{\prime}\right), \quad i=1,2,3
$$

where $g_{\mu}=\left.\left(\partial g\left(v_{i}\right) / \partial v_{i}\right)\right|_{v_{i}=c_{i} v_{i}+\left(1-c_{i}\right) v_{i}^{\prime}, v_{i}^{\prime} \in\left[0, v_{i}\right]}$. By setting $v_{i}^{\prime}=0$, (6) is rewritten as

$$
\text { sat }(v)=H v+d_{s}(v)
$$

where $H=\operatorname{diag}\left\{g_{\mu}\left(v_{1}\right), g_{\mu}\left(v_{2}\right), g_{\mu}\left(v_{3}\right)\right\}$.

The control torque (4) is rewritten as

$$
u=\Gamma v+E d_{s}(v)
$$

where $\Gamma=E H$, and there exists an unknown positive constant $b$ satisfying $0<b \leq\|\Gamma\|<1$.

From (1), (2), and (9), the spacecraft system is further formulated as

$$
\begin{aligned}
& \dot{\sigma}=G(\sigma) \omega \\
& \dot{\omega}=J_{0}^{-1} \Gamma v+F(t)+D(t)
\end{aligned}
$$

where $F(t)=J_{0}^{-1}\left(-\Delta J \dot{\omega}-\omega^{\times} J \omega\right)$ is the uncertainty and $D(t)=$ $J_{0}^{-1}\left(E d_{s}(v)+d\right)$. Because $d_{s}(v)$ and $d$ are bounded, there exists an unknown positive constant $D_{m}$ satisfying $\|D(t)\| \leq D_{m}$.

The matrix $G(\sigma)$ has the following properties [45]:

$$
\begin{aligned}
G(\sigma)^{-1} & =\frac{16}{\left(1+\sigma^{T} \sigma\right)^{2}} G(\sigma)^{T} \\
G(\sigma)^{T} G(\sigma) & =\left(\frac{1+\sigma^{T} \sigma}{4}\right)^{2} I_{3} .
\end{aligned}
$$

From (11) and (12), it is obtained that $1 / 4 \leq\|G\| \leq 1 / 2$.

The control objective in this paper is to develop a fuzzy fault-tolerant finite-time control scheme for the spacecraft with inertia uncertainty, extra disturbance, input saturation, and actuator faults, such that the system output $\sigma$ converges into a small region of the origin within the prescribed bounds in a finite time.

\section{Preliminaries}

In this section, some preliminary knowledge critical for control design and satiability analysis is presented.

3.1. Finite-Time Differentiator. The first-order Levant differentiator [46] is formulated as

$$
\begin{aligned}
\dot{\varphi}_{1} & =\iota \\
\iota & =-\beta_{1}\left|\varphi_{1}-\alpha_{r}\right|^{1 / 2} \operatorname{sgn}\left(\varphi_{1}-\alpha_{r}\right)+\varphi_{2} \\
\dot{\varphi}_{2} & =-\beta_{2} \operatorname{sgn}\left(\varphi_{2}-\dot{\varphi}_{1}\right)
\end{aligned}
$$

where $\alpha_{r}$ is the input signal, $\beta_{1}, \beta_{2}>0$ are the parameters, and $\varphi_{1}$ and $\iota$ are the estimations of $\alpha_{r}$ and $\dot{\alpha}_{r}$, respectively. Following lemma holds if the parameters $\beta_{1}$ and $\beta_{2}$ are chosen properly.

Lemma 1 (see [46]). If the input $\alpha_{r}$ is not affected by the noise, the following equalities are true within a finite time

$$
\begin{gathered}
\varphi_{1}=\alpha_{r}, \\
\iota=\dot{\alpha}_{r} .
\end{gathered}
$$

If the input is affected by noise and satisfying $\left|\alpha_{r}-\alpha_{r}^{\prime}\right| \leq$ $\kappa_{1}$, where $\alpha_{r}^{\prime}$ is the original signal without interference, the following inequalities hold in a finite time:

$$
\begin{gathered}
\left|\varphi_{1}-\alpha_{r}^{\prime}\right| \leq \varsigma_{1} \kappa_{1}=\omega_{1} \\
\left|\iota-\dot{\alpha}_{r}^{\prime}\right| \leq \varsigma_{2} \kappa_{1}^{1 / 2}=\omega_{2}
\end{gathered}
$$

where $\varsigma_{1}$ and $\varsigma_{2}$ are positive constants depended on the design parameters of the differentiator.

3.2. Fuzzy Logic System. A typical fuzzy logic system (FLS) consists of four parts: the fuzzy rules, the fuzzifier, the fuzzy inference engine, and the defuzzifier. The foundation of the FLS is a group of fuzzy If-Then rules as follows:

Rule $l$ : If $x_{1}$ is $F_{1}^{l}$ and $x_{2}$ is $F_{2}^{l}$ and $\ldots$ and $x_{n}$ is $F_{n}^{l}$, Then $y$ is $M^{l}, l=1,2, \ldots, N$.

where $x=\left[x_{1}, x_{2}, \ldots, x_{n}\right]^{T}$ is the input of the FLS, $y$ is the FLS output, $F_{i}^{l}$ and $M^{l}$ denote the fuzzy sets relating to the membership functions $\mu_{G^{l}(y)}$ and $\mu_{F_{i}^{l}}\left(x_{i}\right)$, respectively, and $N$ is the rules number. 
Combining the singleton fuzzifier, center average defuzzification, and product inference, the output of the FLS is obtained as

$$
y(x)=\frac{\sum_{l=1}^{N} \bar{y}_{l} \prod_{i=1}^{n} \mu_{F_{i}^{l}}\left(x_{i}\right)}{\sum_{l=1}^{N}\left[\prod_{i=1}^{n} \mu_{F_{i}^{l}}\left(x_{i}\right)\right]}
$$

where $\bar{y}_{l}=\max _{y \in R} \mu_{G^{l}(y)}$ and $\mu_{F_{i}^{l}}\left(x_{i}\right)$ is the membership function value of the fuzzy variable. The fuzzy basis function is defined as

$$
\Phi_{l}=\frac{\prod_{i=1}^{n} \mu_{F_{i}^{l}}\left(x_{i}\right)}{\sum_{l=1}^{N}\left[\prod_{i=1}^{n} \mu_{F_{i}^{l}}\left(x_{i}\right)\right]}
$$

Define $W=\left[\bar{y}_{1}, \bar{y}_{2}, \ldots, \bar{y}_{N}\right]^{T}=\left[w_{1}, w_{2}, \ldots, w_{3}\right]^{T}$ as the ideal constant weight vector, and the (17) is expressed as

$$
y(x)=W^{T} \Phi(x)
$$

where $\Phi(x)=\left[\Phi_{1}(x), \Phi_{2}(x), \ldots, \Phi_{N}(x)\right]^{T}$ is the basis function vector. The relationship between the FLS and the unknown nonlinear function in the system is given in the following lemma.

Lemma 2. For any continuous function $f(x)$ defined on a compact $\Omega$, then for any constant $\mu>0$, there exists an FLS $W^{T} \Phi(x)$ such that

$$
\sup _{x \in \Omega}\left|f(x)-W^{T} \Phi(x)\right| \leq \mu .
$$

3.3. Prescribed Performance Function. As a priori guaranteeing prescribed behavioral bounds on the output of the system, the prescribed performance function (PPF) is designed as follows:

$$
\rho(t)=\left(\rho_{0}-\rho_{\infty}\right) e^{-\kappa t}+\rho_{\infty}
$$

where $\rho_{0}, \rho_{\infty}$, and $\kappa$ are positive parameters, where $\kappa$ is the prescribed minimum exponential convergence rate and $\rho_{\infty}$ stands for the maximum steady-state error, respectively, and it guarantees the following inequality:

$$
-\underline{\delta}_{i}(t) \rho(t)<\sigma_{i}<\bar{\delta}_{i}(t) \rho(t), \quad i=1,2,3 .
$$

In order to relax the assumption that the initial condition should be precisely known to guarantee the prescribed transient in the classic PPF, e.g., [37], the functions $\underline{\delta}_{i}$ and $\bar{\delta}_{i}$ are satisfying the following properties [47].

(1) $\underline{\delta}_{i}$ and $\bar{\delta}_{i}$ are positive and strictly decreasing; (2) $\lim _{t \rightarrow 0} \underline{\delta}_{i}=+\infty, \lim _{t \rightarrow \infty} \underline{\delta}_{i}=C_{1}, C_{1} \in R^{+}, \lim _{t \rightarrow 0} \bar{\delta}_{i}=+\infty$, and $\lim _{t \rightarrow \infty} \bar{\delta}_{i}=C_{2}, C_{2} \in R^{+}$,

An example of such $\underline{\delta}_{i}$ and $\bar{\delta}_{i}$ is given by

$$
\begin{aligned}
& \dot{\bar{\delta}}_{i}=-p_{i} \bar{\delta}_{i}+q_{i}, \quad p_{i}, q_{i} \in R^{+} \\
& \dot{\delta}_{i}=-a_{i} \underline{\delta}_{i}+b_{i}, \quad a_{i}, b_{i} \in R^{+}
\end{aligned}
$$

where $a_{i}, b_{i}, p_{i}, q_{i}, i=1,2,3$ are positive constants.
For the purpose of designing the control law to guarantee the prescribed performance bounds (22), the error transformations are presented as

$$
\varepsilon_{i}=\frac{1}{2} \ln \left(\frac{\underline{\delta}_{i}(t)+\sigma_{i}(t) / \rho(t)}{\bar{\delta}_{i}(t)-\sigma_{i}(t) / \rho(t)}\right), \quad i=1,2,3 .
$$

Through employing the error transformation (24), the output of the original system $\sigma$ can be guaranteed within the prescribed bound provided that the transformed error $\varepsilon=\left[\varepsilon_{1}, \varepsilon_{2}, \varepsilon_{3}\right]^{T}$ is stable.

Lemma 3 (see [37, 48]). System (10) is invariant under the error transformation (24), and the stabilization of the transformed error $\varepsilon$ can guarantee the output $\sigma$ converge with the prescribed performance described by (22).

The derivative of the $\varepsilon_{i}$ is given as

$$
\dot{\varepsilon}_{i}=r_{i} \dot{\sigma}_{i}+\vartheta_{i}, \quad i=1,2,3
$$

where $r_{i}, \quad i=1,2,3$, is

$$
\begin{aligned}
r_{i} & =\frac{1}{2 \rho(t)}\left(\frac{1}{\sigma_{i}(t) / \rho(t)+\underline{\delta}_{i}(t)}\right. \\
& \left.-\frac{1}{\sigma_{i}(t) / \rho(t)-\bar{\delta}_{i}(t)}\right)
\end{aligned}
$$

and the $\vartheta_{i}, i=1,2,3$, is

$$
\begin{aligned}
\vartheta_{i}= & \frac{\dot{\delta}_{i}(t)-\dot{\rho}(t) \sigma_{i}(t) / \rho^{2}(t)}{2 \sigma_{i}(t) / \rho(t)+\underline{\delta}_{i}(t)} \\
+ & \frac{\dot{\bar{\delta}}_{i}(t)+\dot{\rho}(t) \sigma_{i}(t) / \rho^{2}(t)}{2 \sigma_{i}(t) / \rho(t)-\bar{\delta}_{i}(t)} .
\end{aligned}
$$

Substituting (1) into (25) yields

$$
\dot{\varepsilon}=r G \omega+\vartheta
$$

where $r=\operatorname{diag}\left\{r_{1}, r_{2}, r_{3}\right\}$ and $\vartheta=\left[\vartheta_{1}, \vartheta_{2}, \vartheta_{3}\right]^{T}$.

\section{Main Results}

4.1. Control Design. Define virtual states $z_{1}$ and $z_{2}$ as

$$
\begin{aligned}
& z_{1}=\varepsilon \\
& z_{2}=\omega-x_{c}
\end{aligned}
$$

where $x_{c}=\left[x_{c 1}, x_{c 2}, x_{c 3}\right]^{T}$ is the output of the following finite-time command filter

$$
\begin{aligned}
& \dot{\varphi}_{1 i}=-\beta_{1}\left|\varphi_{1 i}-\alpha_{i}\right|^{1 / 2} \operatorname{sgn}\left(\varphi_{1 i}-\alpha_{i}\right)+\varphi_{2 i} \\
& \dot{\varphi}_{2 i}=-\beta_{2} \operatorname{sgn}\left(\varphi_{2 i}-\dot{\varphi}_{1 i}\right),
\end{aligned}
$$


where the input $\alpha=\left[\alpha_{1}, \alpha_{2}, \alpha_{3}\right]^{T}$ is the virtual control to be designed later and the output $x_{c i}=\varphi_{1 i}, i=1,2,3$.

Based on the command filtered backstepping control approach, the compensated stabilization errors are given by $s_{1}=z_{1}-\xi_{1}, s_{2}=z_{2}-\xi_{2}$, where $\xi_{1}$ and $\xi_{2}$ are the error compensating signals to reduce the influence of $x_{c}-\alpha$.

Choose the Lyapunov function as $V_{1}=(1 / 2) s_{1}^{T} s_{1}$. Using (28), the time derivative of $V_{1}$ is

$$
\begin{aligned}
\dot{V}_{1} & =s_{1}^{T} \dot{s}_{1}=s_{1}^{T}\left(\dot{z}_{1}-\dot{\xi}_{1}\right)=s_{1}^{T}\left(r G \omega+\vartheta-\dot{\xi}_{1}\right) \\
& =s_{1}^{T}\left[r G\left(z_{2}+x_{c}\right)+\vartheta-\dot{\xi}_{1}\right] \\
& =s_{1}^{T}\left[r G z_{2}+r G \alpha+r G\left(x_{c}-\alpha\right)+\vartheta-\dot{\xi}_{1}\right] .
\end{aligned}
$$

The virtual control $\alpha$ and error compensation $\xi_{1}$ are designed as

$$
\alpha=G^{-1} r^{-1}\left[-k_{1} z_{1}-\tau_{1} \operatorname{sig}^{\gamma}\left(s_{1}\right)-\vartheta\right]
$$

and

$$
\dot{\xi}_{1}=-k_{1} \xi_{1}+r G\left(x_{c}-\alpha\right)+r G \xi_{2}-l_{1} \operatorname{sig}\left(\xi_{1}\right)
$$

where $k_{1}, \tau_{1}, l_{1}>0,0<\gamma<1$ are design parameters, $\operatorname{sig}^{\gamma}\left(s_{1}\right)$ $=\left[\left|s_{11}\right|^{\gamma} \operatorname{sgn}\left(s_{11}\right),\left|s_{12}\right|^{\gamma} \operatorname{sgn}\left(s_{12}\right),\left|s_{13}\right|^{\gamma} \operatorname{sgn}\left(s_{13}\right)\right]^{T}$, and $\operatorname{sig}\left(\xi_{1}\right)=$ $\left[\operatorname{sgn}\left(\xi_{11}\right), \operatorname{sgn}\left(\xi_{12}\right), \operatorname{sgn}\left(\xi_{13}\right)\right]^{T}$ with $\xi_{1 i}(0)=0, i=1,2,3$.

Substituting (32) and (33) into (31) yields

$$
\dot{V}_{1}=s_{1}^{T}\left[-k_{1} s_{1}+r G s_{2}-\tau_{1} \operatorname{sig}^{\gamma}\left(s_{1}\right)+l_{1} \operatorname{sig}\left(\xi_{1}\right)\right] .
$$

Construct the second Lyapunov function as

$$
V_{2}=V_{1}+\frac{1}{2} s_{2}^{T} s_{2} .
$$

Taking the derivative of $V_{2}$ along with (10) and (29) yields

$$
\begin{aligned}
\dot{V}_{2} & =\dot{V}_{1}+s_{2}^{T} \dot{s}_{2}=\dot{V}_{1}+s_{2}^{T}\left(\dot{\omega}-\dot{x}_{c}-\dot{\xi}_{2}\right) \\
& =\dot{V}_{1}+s_{2}^{T}\left(J_{0}^{-1} \Gamma v+F+D-\dot{x}_{c}-\dot{\xi}_{2}\right) .
\end{aligned}
$$

The error compensation is designed as $\xi_{2}=\dot{\xi}_{2}=[0,0$, $0]^{T}$. Let $\bar{F}=F-\dot{x}_{c}+r G s_{1}+s_{2}$. Substituting (34) into (36) and using the fact that $G$ is symmetric lead to

$$
\begin{aligned}
\dot{V}_{2}= & \dot{V}_{1}+s_{2}^{T}\left(\bar{F}-r G s_{1}-s_{2}+J_{0}^{-1} \Gamma v+D\right) \\
= & -k_{1} s_{1}^{T} s_{1}-\tau_{1} \sum_{1}^{3}\left|s_{1 i}\right|^{\gamma+1}+s_{1}^{T} l_{1} \operatorname{sig}\left(\xi_{1}\right)-s_{2}^{T} s_{2} \\
& +s_{2}^{T}\left(\bar{F}+J_{0}^{-1} \Gamma v+D\right) .
\end{aligned}
$$

The fuzzy logic systems (19) are utilized to approximate the unknown nonlinear $\bar{F}$. From Lemma 2, for any given constant $\mu>0$, there always exists an FLS such that

$$
\bar{F}_{i}=W_{i}^{T} \Phi_{i}\left(Z_{n}\right)+\delta_{i}, \quad i=1,2,3
$$

where $\Phi_{i}=\left[\Phi_{i 1}, \Phi_{i 2}, \ldots, \Phi_{i N}\right]^{T}$ is the basis function vector, the approximation error $\delta_{i}$ satisfied $\left|\delta_{i}\right| \leq \mu$, and $Z_{n}=$ $\left[\omega^{T}, x_{c}^{T}, \dot{x}_{c}^{T}\right]^{T}$. hold:

By Young's inequality and (38), the following inequalities

$$
\begin{aligned}
s_{2}^{T} \bar{F} & \leq \sum_{i=1}^{3} s_{2 i} W_{i}^{T} \Phi_{i}+\sum_{i=1}^{3} s_{2 i} \mu \\
& \leq \frac{\theta \sum_{i=1}^{3} b s_{2 i} \Phi_{i}^{T} \Phi_{i}}{2 h^{2}}+\frac{3 h^{2}}{2}+\frac{\left\|s_{2}\right\|^{2}}{2}+\frac{3 \mu^{2}}{2} \\
s_{2}^{T} D & \leq \frac{\left\|s_{2}\right\|^{2}}{2}+\frac{D_{m}^{2}}{2} \\
s_{1}^{T} l_{1} \operatorname{sig}\left(\xi_{1}\right) & \leq \frac{l_{1}\left\|s_{1}\right\|^{2}}{2}+\frac{l_{1}}{2}
\end{aligned}
$$

where $\theta=(1 / b) \max \left\{\left\|W_{1}\right\|^{2},\left\|W_{2}\right\|^{2},\left\|W_{3}\right\|^{2}\right\}$.

The commanded controller is designed as

$$
v=J_{0}\left[-k_{2} s_{2}-\frac{\hat{\theta} \eta s_{2}}{2 h^{2}}-\tau_{2} \operatorname{sig}^{\gamma}\left(s_{2}\right)\right]
$$

where $k_{2}, \tau_{2}, h$ are positive design parameters, $\eta=$ $\operatorname{diag}\left\{\Phi_{1}^{T} \Phi_{1}, \Phi_{2}^{T} \Phi_{2}, \Phi_{3}^{T} \Phi_{3}\right\}$, adn $\hat{\theta}$ is the estimation of $\theta$.

It is obtained for the commanded controller (42) that

$$
\begin{aligned}
s_{2}^{T} J_{0}^{-1} \Gamma v= & s_{2}^{T}\left[-k_{2} \Gamma s_{2}-\frac{\Gamma \hat{\theta} \eta s_{2}}{2 h^{2}}-\tau_{2} \Gamma \operatorname{sig}^{\gamma}\left(v_{2}\right)\right] \\
\leq & -k_{2} b s_{2}^{T} s_{2}-\frac{\widehat{\theta} \sum_{i=1}^{3} b s_{2 i}^{2} \Phi_{i}{ }^{T} \Phi_{i}}{2 h^{2}} \\
& -\tau_{2} b \sum_{i=1}^{3}\left|s_{2 i}\right|^{\gamma+1} .
\end{aligned}
$$

Using (39)-(43), the derivative of the $V_{2}$ is simplified as

$$
\begin{aligned}
\dot{V}_{2} \leq & -\left(k_{1}-0.5 l_{1}\right) s_{1}^{T} s_{1}-\tau_{1} \sum_{i=1}^{3}\left|s_{1 i}\right|^{\gamma+1}-k_{2} b s_{2}^{T} s_{2} \\
& -\tau_{2} b \sum_{i=1}^{3}\left|s_{2 i}\right|^{\gamma+1}+\frac{(\theta-\hat{\theta}) \sum_{i=1}^{3} b s_{2 i}^{2} \Phi_{i}{ }^{T} \Phi_{i}}{2 h^{2}} \\
& +\frac{3 h^{2}}{2}+\frac{3 \mu^{2}}{2}+\frac{D_{m}^{2}}{2}+\frac{l_{1}}{2} .
\end{aligned}
$$

The update law of $\widehat{\theta}$ is designed as

$$
\dot{\hat{\theta}}=\frac{\lambda \sum_{i=1}^{3} s_{2 i}^{2} \Phi_{i}^{T} \Phi_{i}}{2 h^{2}}-m_{1} \hat{\theta}
$$

where $\lambda$ and $m_{1}$ are positive design parameters.

4.2. Stability Analysis. Before providing the stability analysis, the following two lemmas are given.

Lemma 4 (see [49]). For $0<a<1$ and $x_{i} \in \mathfrak{R}, i=1,2,3 \ldots$, the following inequality holds:

$$
\sum_{i=1}^{3}\left|x_{i}\right|^{a+1} \geq\left(\sum_{i=1}^{3}\left|x_{i}\right|^{2}\right)^{(a+1) / 2} .
$$


Lemma 5 (see [50]). For any real number $\left.0<\gamma<1, \lambda_{1}, \lambda_{2}\right\rangle$ 0 , an continuous positive-definite Lyapunov function $V(x)$ satisfied the from as $\dot{V}(x)+\lambda_{1} V(x)+\lambda_{2} V^{\gamma}(x) \leq 0$, then $V \equiv 0$ can be achieved in a finite time and the setting time can be estimated by

$$
T_{\text {reach }} \leq \frac{1}{\lambda_{1}(1-\gamma)} \ln \frac{\lambda_{1} V^{1-\gamma}(0)+\lambda_{2}}{\lambda_{2}}
$$

where $V(0)$ is the initial value of $V(x)$.

Theorem 6. Consider the spacecraft stabilization system described in (1) and (2) subject to input saturation and actuator fault (4) with finite-time command filter (30), the virtual control (32), the error compensation (33), the controller (42), and the update law (45), and then

(i) the transformed error $\varepsilon$ converges into a small region of the origin in a finite time, and $\omega$ is bounded in a finite time;

(ii) the prescribed control performance of $\sigma$ (22) is preserved.

Proof. Construct the Lyapunov function as

$$
V=V_{2}+\frac{b}{2 \lambda} \widetilde{\theta}^{2}
$$

where $\tilde{\theta}=\theta-\widehat{\theta}$.

According to (44), the time derivative of $V$ is

$$
\begin{aligned}
\dot{V} \leq & -\left(k_{1}-0.5 l_{1}\right) s_{1}^{T} s_{1}-\tau_{1} \sum_{i=1}^{3}\left|s_{1 i}\right|^{\gamma+1}-k_{2} b s_{2}^{T} s_{2} \\
& -\tau_{2} b \sum_{i=1}^{3}\left|s_{2 i}\right|^{\gamma+1}+\frac{\sum_{i=1}^{3} s_{2 i}^{2} \Phi_{i}^{T} \Phi_{i}}{2 h^{2}} b \widetilde{\theta}+\frac{3 h^{2}}{2} \\
& +\frac{3 \mu^{2}}{2}+\frac{D_{m}^{2}}{2}+\frac{l_{1}}{2}-\frac{b \tilde{\dot{\theta} \theta}}{\lambda} .
\end{aligned}
$$

Substituting update law (45) into (49) yields

$$
\begin{aligned}
\dot{V} \leq & -\left(k_{1}-0.5 l_{1}\right) s_{1}^{T} s_{1}-\tau_{1} \sum_{i=1}^{3}\left|s_{1 i}\right|^{\gamma+1}-k_{2} b s_{2}^{T} s_{2} \\
& -\tau_{2} b \sum_{i=1}^{3}\left|s_{2 i}\right|^{\gamma+1}+\frac{3 h^{2}}{2}+\frac{3 \mu^{2}}{2}+\frac{D_{m}^{2}}{2}+\frac{l_{1}}{2} \\
& +\frac{m_{1} b \tilde{\theta} \hat{\theta}}{\lambda} .
\end{aligned}
$$

According to Young's inequality, the following inequality is hold:

$$
\frac{m_{1} b \widetilde{\theta} \widehat{\theta}}{\lambda} \leq \frac{-3 m_{1} b \widetilde{\theta}^{2}}{4 \lambda}+\frac{m_{1} b \theta^{2}}{\lambda} .
$$

Considering $0<\gamma<1$, it is concluded that

$$
\left(\frac{m_{1} b \widetilde{\theta}^{2}}{2 \lambda}\right)^{(\gamma+1) / 2}-\frac{m_{1} b \widetilde{\theta}^{2}}{2 \lambda} \leq \frac{1}{4} .
$$

Using (51), (52), and Lemma 4, the time derivative of $V$ is expressed as

$$
\dot{V} \leq-\left(k_{1}-0.5 l_{1}\right) s_{1}^{T} s_{1}-\tau_{1}\left(\sum_{1}^{3}\left|s_{1 i}\right|^{2}\right)^{(\gamma+1) / 2}
$$

$$
\begin{aligned}
& -k_{2} b s_{2}^{T} s_{2}-\tau_{2} b\left(\sum_{1}^{3}\left|s_{2 i}\right|^{2}\right)^{(\gamma+1) / 2}-\frac{m_{1} b \widetilde{\theta}^{2}}{4 \lambda} \\
& -\left(\frac{m_{1} b \widetilde{\theta}^{2}}{2 \lambda}\right)^{(\gamma+1) / 2}+\frac{3 h^{2}}{2}+\frac{3 \mu^{2}}{2}+\frac{D_{m}^{2}}{2}+\frac{l_{1}}{2} \\
& +\frac{m_{1} b \theta^{2}}{\lambda}+\frac{1}{4}
\end{aligned}
$$

which leads to

$$
\dot{V} \leq-\lambda_{1} V-\lambda_{2} V^{(\gamma+1) / 2}+\mu_{1}
$$

where $\lambda_{1}=\min \left\{2 k_{1}-l_{1}, 2 k_{2} b, 0.5 m_{1}\right\}, \lambda_{2}=\min \left\{2^{(\gamma+1) / 2} \tau_{1}\right.$, $\left.2^{(\gamma+1) / 2} \tau_{2} b, m_{1}^{(\gamma+1) / 2}\right\}$, and $\mu_{1}=3 h^{2} / 2+3 \mu^{2} / 2+D_{m}^{2} / 2+$ $l_{1} / 2+m_{1} b \theta^{2} / \lambda+1 / 4$. According to Lemma 5 , it is concluded that $s_{1}$ and $s_{2}$ converge to the small region $\left\|s_{i}\right\| \leq$ $\max \left\{\sqrt{2 \mu_{1} / \lambda_{1}}, \sqrt{2\left(\mu_{1} / \lambda_{2}\right)^{2 /(\gamma+1)}}\right\}, i=1,2$, in a finite time $T_{1} \leq$ $\left(1 / \lambda_{1}(1-\gamma)\right) \ln \left(\left(\lambda_{1} V^{1-\gamma}(0)+\lambda_{2}\right) / \lambda_{2}\right)$. From the definition $s_{1}=\varepsilon-\xi_{1}, s_{2}=z_{2}-\xi_{2}$, if the finite-time convergence of the $\xi_{i}$ is guaranteed, then it is confirmed that the states $z_{1}$ and $z_{2}$ can converge into a small neighbourhood of the origin within a finite time.

Since $\xi_{2}=\dot{\xi}_{2}=[0,0,0]^{T}$ is given in the control design, in order to show that $\xi_{1}$ is bounded in a finite time, the following Lyapunov function is chosen:

$$
V_{3}=\frac{1}{2} \xi_{1}^{T} \xi_{1}
$$

Differentiating $V_{3}$ along with (33) yields

$$
\begin{aligned}
\dot{V}_{3} & =\xi_{1}^{T} \dot{\xi}_{1} \\
& =\xi_{1}^{T}\left[-k_{1} \xi_{1}+G\left(x_{c}-\alpha\right)+G \xi_{2}-l_{1} \operatorname{sig}\left(\xi_{1}\right)\right] \\
& =-k_{1} \xi_{1}^{T} \xi_{1}-l_{1} \sum_{1}^{3}\left|\xi_{i}\right|+\xi_{1}^{T} G \xi_{2}+\xi_{1}^{T} G\left(x_{c}-\alpha\right) .
\end{aligned}
$$

According to the Lemma $1,\left\|x_{c}-\alpha\right\| \leq \Phi$ can be achieved in a finite time $T_{2}$, and combining $\|G\| \leq 1 / 2$, the following inequality is obtained:

$$
\xi_{1}^{T} G\left(x_{c}-\alpha\right) \leq \frac{1}{2} \omega \sum_{1}^{3}\left|\xi_{i}\right| .
$$

Substituting (57) and $\xi_{2}=[0,0,0]^{T}$ into (56) leads to

$$
\begin{aligned}
\dot{V}_{3} & \leq-k_{1} \xi_{1}^{T} \xi_{1}-\left(l_{1}-\frac{1}{2} \omega\right)\left(\sum_{1}^{3}\left|\xi_{i}\right|^{2}\right)^{1 / 2} \\
& \leq-2 k_{1} V_{3}-\sqrt{2}\left(l_{1}-\frac{1}{2} \omega\right) V_{3}^{1 / 2} .
\end{aligned}
$$

According to Lemma 5, it is illustrated that $\xi_{1}$ can converge to the origin in the finite time $T_{3}$ by choosing suitable parameter satisfying $l_{1}>(1 / 2) \omega$. Since $z_{1}=s_{1}+\xi_{1}$ and $z_{2}=s_{2}+\xi_{2}$, the transformed error $\varepsilon=z_{1}$ converges to a 
TABLE 1: Parameters of the control scheme.

\begin{tabular}{lccccc}
\hline Parameter & Value & Parameter & Value & Parameter & Value \\
\hline$k_{1}$ & 0.1 & $\beta_{1}$ & 3 & $p_{1}, q_{1}$ & 5,1 \\
$k_{2}$ & 0.8 & $\beta_{2}$ & 4 & $a_{1}, b_{1}$ & 5,4 \\
$l_{1}$ & 0.01 & $h$ & 0.5 & $p_{2}, q_{2}$ & 5,1 \\
$\tau_{1}$ & 0.01 & $m_{1}$ & 0.001 & $a_{2}, b_{2}$ & 5,5 \\
$\tau_{2}$ & 0.5 & $\rho_{0}$ & 0.5 & $p_{3}, q_{3}$ & 5,4 \\
$\gamma$ & 0.3 & $\rho_{\infty}$ & 0.001 & $a_{3}, b_{3}$ & 5,1 \\
$\lambda$ & 2 & $\kappa$ & 0.1 & & \\
\hline
\end{tabular}

small region of the origin within the finite time $T_{F}=T_{1}+T_{2}+$ $T_{3}$, and from the definition $\omega=z_{2}+x_{c}$, $\omega$ is also bounded. According to Lemma 3, the stabilization of transformed error $\varepsilon$ is sufficient to guarantee $\sigma$ converge with prescribed performance described by (22). This completes the proof.

Remark 7. From (54) and (58), it is seen that the increase of $k_{1}, k_{2}, \tau_{1}, \tau_{2}, l_{1}$ and decrease of $\gamma$ lead to better convergence speed, but large $\tau_{1}, l_{1}$ and small $\gamma$ will result in chattering problem. Consequently, the choice of the parameters $\tau_{1}, l_{1}$, and $\gamma$ should be considered with a trade between the convergence speed and chattering reduction.

\section{Simulation}

In order to illustrate the effectiveness of the proposed control scheme, the simulation results and discussions are presented in this section. The spacecraft model is expressed as (1) and (2) where the initial values of the state parameters are set as

$$
\begin{aligned}
\sigma(0) & =[-0.3,-0.4,0.2]^{T} \\
\omega(0) & =[0,0,0]^{T} \mathrm{rad} / \mathrm{s} .
\end{aligned}
$$

The nominal inertia matrix is

$$
J_{0}=\left[\begin{array}{ccc}
350 & 3 & 4 \\
3 & 270 & 10 \\
4 & 10 & 192
\end{array}\right]
$$

and the uncertainty $\Delta J$ is

$$
\begin{aligned}
\Delta J= & \operatorname{diag}\{5 \sin (0.1 t), 7 \sin (0.2 t), 9 \sin (0.3 t)\} \mathrm{kg} \\
& \cdot \mathrm{m}^{2} .
\end{aligned}
$$

The external disturbance is

$$
\text { d }
$$$$
=\left(\|\omega\|^{2}+0.05\right)[\sin (0.8 t), \cos (0.5 t), \sin (0.3 t)]^{T} \mathrm{~N}
$$

$\cdot \mathrm{m}$.

The maximum torque of the actuators is $v_{M i}=8 \mathrm{~N} \cdot \mathrm{m}$, $i=1,2,3$. In order to reflect the fault condition of real actuators such as flywheels, a time-varying loss of actuator effectiveness fault $E=\operatorname{diag}\left\{E_{1}, E_{2}, E_{3}\right\}$ is given as [9]

$$
\begin{array}{ll}
E_{1}=0.2+0.1 \sin (0.2 \pi t), & t \geq 15 \mathrm{~s} \\
E_{2}=0.2+0.1 \sin (0.3 \pi t), & t \geq 12 \mathrm{~s} \\
E_{3}=0.2+0.1 \sin (0.4 \pi t), & t \geq 10 \mathrm{~s} .
\end{array}
$$

In practice, to perform a high-precision stabilization and safety during the maneuvers, the state $\sigma_{i}$ should be stabilize to a small region, i.e., $\left|\sigma_{i}\right| \leq 1 \times 10^{-3}, i=1,2,3$, and no overshoot is allowed.

The comparative simulations are given to verify the effectiveness of the proposed control scheme. For the notation convenience, the three compared control schemes are given as follows.

M1: the proposed control scheme including the finitetime command filter (30), virtual control (32), error compensation (33), commanded controller (42), and the update law (45). The control parameter settings are shown in Table 1.

M2: the conventional backstepping control scheme [26]. The virtual control $\alpha$, commanded controller $v$, and fuzzy adaptive update law are given by

$$
\begin{aligned}
\alpha & =-k_{1} G^{-1} z_{1} \\
\nu & =J_{0}\left(-k_{2} z_{2}-\frac{\hat{\theta} \eta s_{2}}{2 h^{2}}\right) \\
\dot{\hat{\theta}} & =\frac{\lambda \sum_{i=1}^{3} s_{2 i}^{2} \Phi_{i}{ }^{T} \Phi_{i}}{2 h^{2}}-m_{1} \hat{\theta}
\end{aligned}
$$

where $z_{1}=\sigma, z_{2}=\omega-\alpha$, and the control parameters $k_{1}, k_{2}, \lambda, h$ are set the same as M1 scheme.

M3: the adaptive fast terminal sliding mode control scheme proposed in [22]. The sliding function $S$ is given by

$$
S=\omega+K_{1} \sigma+K_{2} S_{a u}
$$

where $K_{1}, K_{2}$ are design parameters and $S_{a u}=\left[S_{a u 1}, S_{a u 2}\right.$, $\left.S_{a u 3}\right]^{T}$ is

$$
\begin{aligned}
& S_{\text {aui }} \\
& = \begin{cases}\operatorname{sgn}\left(\sigma_{i}\right)\left|\delta_{i}\right|^{r}, & \text { if }\left|\sigma_{i}\right| \geq \varepsilon \\
(2-r) \varepsilon^{r-1} \sigma_{i}+(2-r) \varepsilon^{r-2} \operatorname{sgn}\left(\sigma_{i}\right) \sigma_{i}^{2}, & \text { if }\left|\sigma_{i}\right|<\varepsilon\end{cases}
\end{aligned}
$$

where $\varepsilon>0$ is a small constant scalar; $0<r<1$ is a design parameter.

The control law is

$$
v=-\tau S-\rho \operatorname{sig}^{0.5}(S)-v_{s s}(t)
$$

where $\tau, \rho$ are design parameters, $\operatorname{sig}^{0.5}(S)=\left[\left|S_{1}\right|^{0.5} \operatorname{sgn}\left(S_{1}\right)\right.$, $\left.\left|S_{2}\right|^{0.5} \operatorname{sgn}\left(S_{2}\right),\left|S_{3}\right|^{0.5} \operatorname{sgn}\left(S_{3}\right)\right]^{T}$, and $v_{s s}(t)$ is

$$
v_{s s}(t)
$$



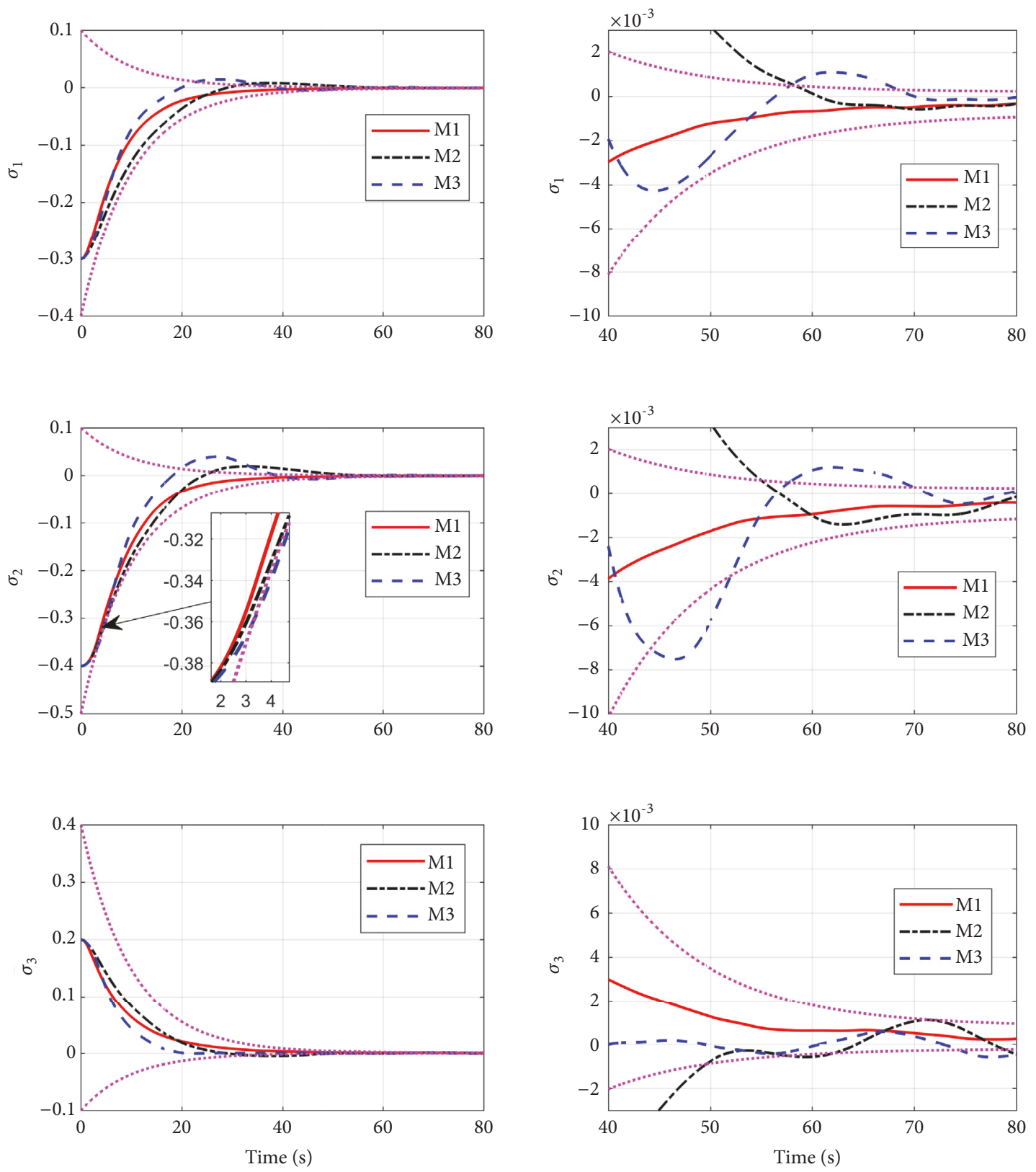

FIGURE 1: Attitude $\sigma$ along with corresponding performance bounds.

$$
= \begin{cases}\frac{S}{\|S\|} \widehat{F}(t)+\frac{S}{\|S\|^{2}} \sum_{i=1}^{3} \frac{o_{i}}{4 p_{i}} \widehat{c}_{i}(t), & \text { if }\|S\| \widehat{F}(t)>\epsilon \\ \frac{S}{\epsilon} \widehat{F}^{2}(t), & \text { if }\|S\| \widehat{F}(t) \leq \epsilon\end{cases}
$$

where $\epsilon>0$ is a small constant scalar, $\widehat{F}(t)=\widehat{c}_{1}(t)+\widehat{c}_{2}(t)\|\omega\|+$ $\widehat{c}_{3}(t)\|\omega\|^{2}$, and the update laws are given by

$$
\dot{\widehat{c}}_{1}=-o_{1} \widehat{c}_{1}+p_{1}\|S\|
$$

$$
\begin{aligned}
& \dot{\vec{c}}_{2}=-o_{2} \widehat{c}_{2}+p_{2}\|S\|\|\omega\| \\
& \dot{\widehat{c}}_{3}=-o_{3} \widehat{c}_{3}+p_{3}\|S\|\|\omega\|^{2}
\end{aligned}
$$

where $o_{i}, p_{i}, i=1,2,3$, are design parameters. The parameters are chosen as $r=9 / 11, K_{1}=0.4 I_{3}, K 2=0.2 I_{3}, \tau=10 I_{3}$, $\rho=1, o_{i}=0.1, p_{i}=2, i=1,2,3, \epsilon=0.01$, and $\varepsilon=0.0001$.

The simulation results are shown in Figures $1-5$. The attitude described by MRPs $\sigma_{i}(t), i=1,2,3$, along with the 

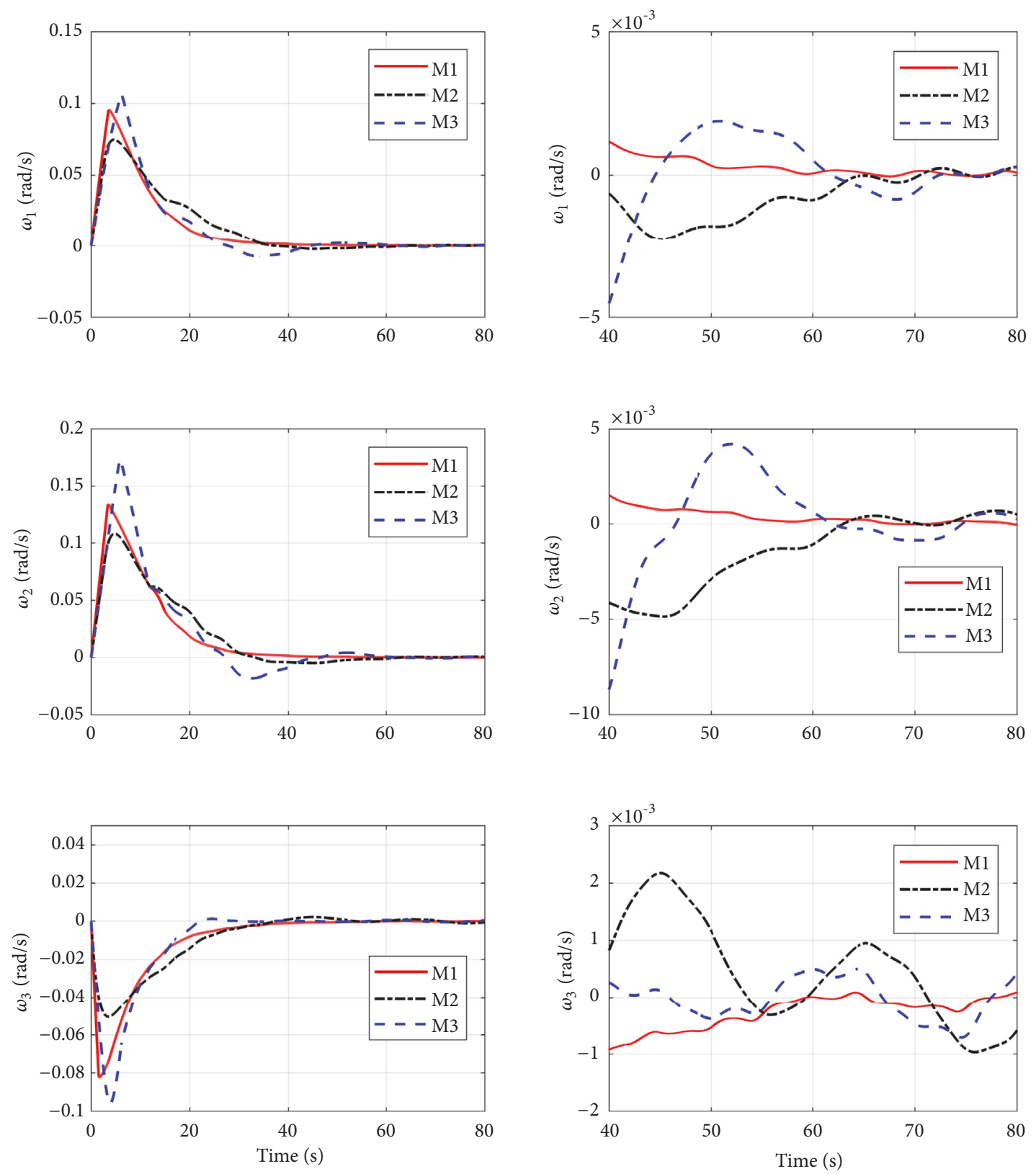

FIgURE 2: Angular velocity $\omega$.

corresponding performance bounds is depicted in Figure 1. As shown in Figure 1, although all the three schemes could achieve the attitude stabilization, the M2 and M3 fail to meet the prescribed transient and steady-state error bound, while the proposed M1 scheme remains in the prescribed bound all the time. The angular velocities $\omega$ of the three schemes are depicted in Figure 2, which shows that M1 can provide better angular velocity performance than M2 and M3. From Figures 1 and 2, it is concluded that the attitude stabilization with prescribed performance and high precision is achieved in a finite time with the proposed control scheme. Figure 3 shows the actual control signals $u$, which are quite similar for different three schemes. The three-dimensional trajectory of the transformed error $\varepsilon(t)$ is shown in Figure 4; it can be seen that the transformed error can converge to the neighbourhood of the origin in a finite time (around $40 \mathrm{~s}$ ) subject to the actuator fault and input saturation. The convergence performance of the estimated parameter $\hat{\theta}$ is shown in Figure 5, and it is clear that the parameter $\widehat{\theta}$ converges to a positive constant. From Figures 

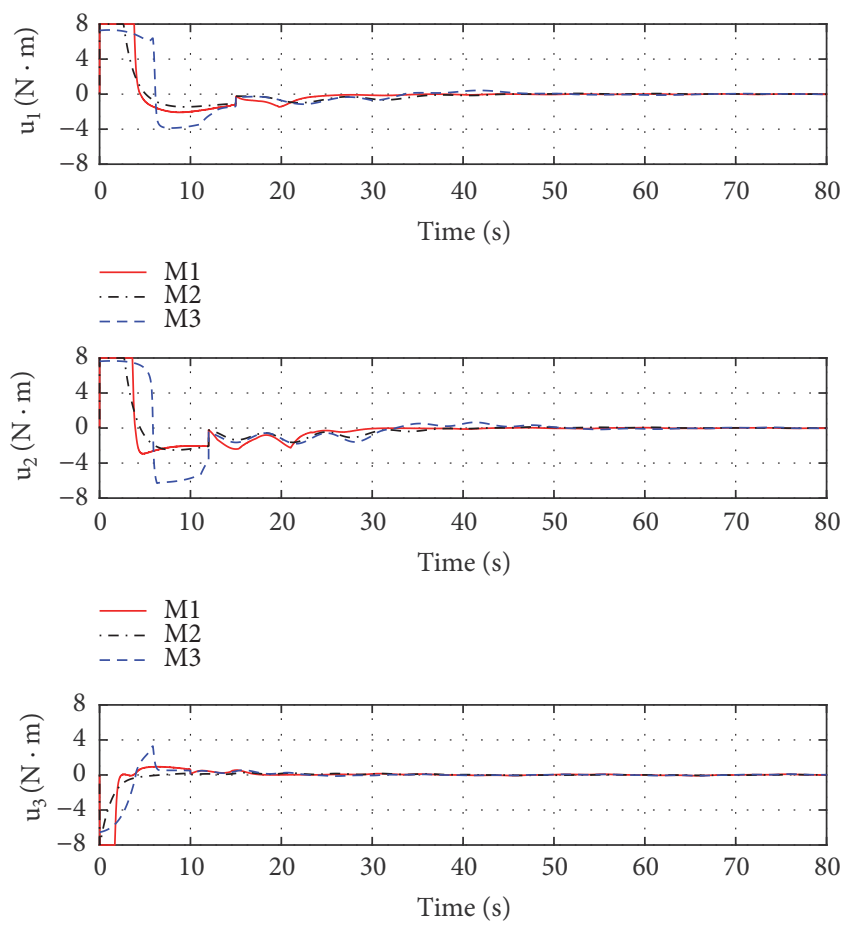

$\begin{array}{ll}- & \text { M1 } \\ -- & \text { M2 } \\ --- & \text { M3 }\end{array}$

FIgure 3: Actual Control torque $u$.

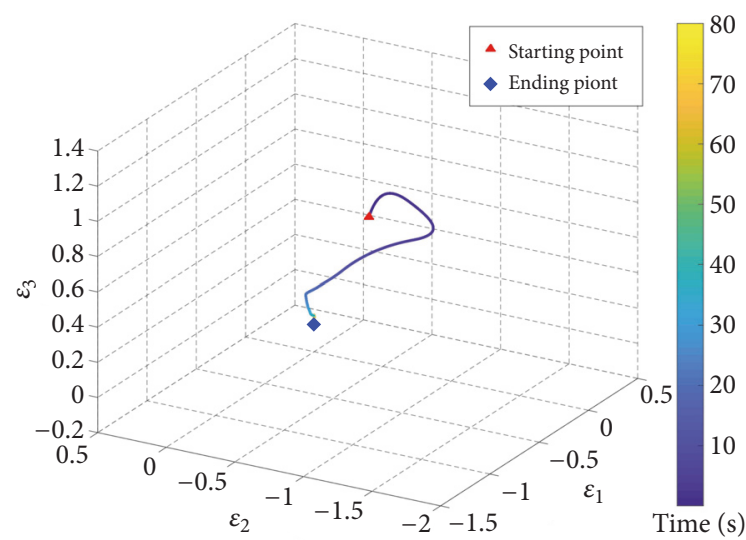

FIGURE 4: Three-dimensional trajectory of the transformed error $\varepsilon(t)$.

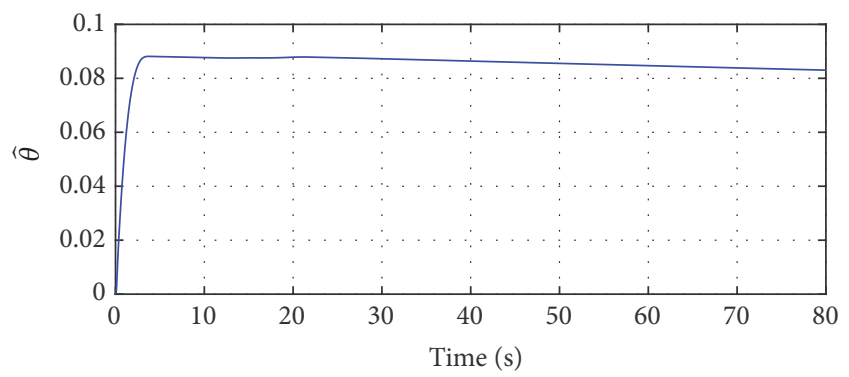

FIGURE 5: Estimated parameter $\widehat{\theta}$.
$1-5$, it is concluded that the proposed control scheme can achieve the prescribed performance within a finite time in the presence of the inertia uncertainty, external disturbance, actuator saturation, and faults.

\section{Conclusion}

The problem of attitude stabilization with guaranteed transient and steady-state performance has been investigated in this paper for the spacecraft systems with inertia uncertainty, external disturbance, actuator saturation, and faults. The singularity problem caused by the differentiation of the virtual control is avoided by the proposed first-order command filter. Then, incorporate the prescribed performance boundary into the controller design by using the error transformation to guarantee the prescribed performance of the system output. The control scheme stabilizes the system within a finite time with the proposed adaptive finite-time fault-tolerant control scheme. Finally, simulation results have been provided to verify the effectiveness of the proposed control algorithm.

\section{Data Availability}

The data used to support the findings of this study are available from the corresponding author upon request.

\section{Conflicts of Interest}

The authors declare that they have no conflicts of interest.

\section{Acknowledgments}

This work was supported by National Natural Science Foundation of China (Projects Nos. 61473262, 61573320, and 61403343) and Zhejiang Provincial Natural Science Foundation (No. LY17F030018).

\section{References}

[1] D. Thakur, S. Srikant, and M. R. Akella, "Adaptive attitudetracking control of spacecraft with uncertain time-varying inertia parameters," Journal of Guidance, Control, and Dynamics, vol. 38, no. 1, pp. 41-52, 2015.

[2] K. Lu, Y. Xia, Z. Zhu, and M. V. Basin, "Sliding mode attitude tracking of rigid spacecraft with disturbances," Journal of The Franklin Institute, vol. 349, no. 2, pp. 413-440, 2012.

[3] I. Ali, G. Radice, and J. Kim, "Backstepping control design with actuator torque bound for spacecraft attitude maneuver," Journal of Guidance, Control, and Dynamics, vol. 33, no. 1, pp. 254-259, 2010.

[4] W. Luo, Y.-C. Chu, and K.-V. Ling, "Ho inverse optimal attitude-tracking control of rigid spacecraft," Journal of Guidance, Control, and Dynamics, vol. 28, no. 3, pp. 481-493, 2005.

[5] Z. Zhu, Y. Xia, and M. Fu, "Attitude stabilization of rigid spacecraft with finite-time convergence," International Journal of Robust and Nonlinear Control, vol. 21, no. 6, pp. 686-702, 2011.

[6] H. Du, S. Li, and C. Qian, "Finite-time attitude tracking control of spacecraft with application to attitude synchronization," IEEE 
Transactions on Automatic Control, vol. 56, no. 11, pp. 2711-2717, 2011.

[7] S. Li, H. Du, and P. Shi, "Distributed attitude control for multiple spacecraft with communication delays," IEEE Transactions on Aerospace and Electronic Systems, vol. 50, no. 3, pp. 1765-1773, 2014.

[8] Z. Song, C. Duan, J. Wang, and Q. Wu, "Chattering-free fullorder recursive sliding mode control for finite-time attitude synchronization of rigid spacecraft," Journal of The Franklin Institute, 2018.

[9] B. Xiao, Q. Hu, and M. I. Friswell, "Active fault-tolerant attitude control for flexible spacecraft with loss of actuator effectiveness," International Journal of Adaptive Control and Signal Processing, vol. 27, no. 11, pp. 925-943, 2013.

[10] A.-M. Zou and K. D. Kumar, "Adaptive fuzzy fault-tolerant attitude control of spacecraft," Control Engineering Practice, vol. 19, no. 1, pp. 10-21, 2011.

[11] S. Varma and K. D. Kumar, "Fault tolerant satellite attitude control using solar radiation pressure based on nonlinear adaptive sliding mode," Acta Astronautica, vol. 66, no. 3-4, pp. 486-500, 2010.

[12] Q. Hu and X. Shao, "Smooth finite-time fault-tolerant attitude tracking control for rigid spacecraft," Aerospace Science and Technology, vol. 55, pp. 144-157, 2016.

[13] A. H. J. De Ruiter, "Adaptive spacecraft attitude control with actuator saturation," Journal of Guidance, Control, and Dynamics, vol. 33, no. 5, pp. 1692-1696, 2010.

[14] Z. Zhu, Y. Q. Xia, and M. Y. Fu, "Adaptive sliding mode control for attitude stabilization with actuator saturation," IEEE Transactions on Industrial Electronics, vol. 58, no. 10, pp. 48984907, 2011.

[15] K. Lu, Y. Xia, and M. Fu, "Controller design for rigid spacecraft attitude tracking with actuator saturation," Information Sciences, vol. 220, pp. 343-366, 2013.

[16] Q. Shen, D. Wang, S. Zhu, and E. K. Poh, "Finite-time faulttolerant attitude stabilization for spacecraft with actuator saturation," IEEE Transactions on Aerospace and Electronic Systems, vol. 51, no. 3, pp. 2390-2405, 2015.

[17] B. Xiao, Q. Hu, Y. Zhang, and X. Huo, "Fault-tolerant tracking control of spacecraft with attitude-only measurement under actuator failures," Journal of Guidance, Control, and Dynamics, vol. 37, no. 3, pp. 838-849, 2014.

[18] Q. Chen, S. Xie, M. Sun, and X. He, "Adaptive non-singular fixed-time attitude stabilization of uncertain spacecraft," IEEE Transactions on Aerospace and Electronic Systems, 2018.

[19] H. B. Du and S. H. Li, "Finite-time attitude stabilization for a spacecraft using homogeneous method," Journal of Guidance, Control, and Dynamics, vol. 35, no. 3, pp. 740-748, 2012.

[20] H. Gui, L. Jin, and S. Xu, "Simple finite-time attitude stabilization laws for rigid spacecraft with bounded inputs," Aerospace Science and Technology, vol. 42, pp. 176-186, 2015.

[21] K. Lu and Y. Xia, "Finite-time attitude stabilization for rigid spacecraft," International Journal of Robust and Nonlinear Control, vol. 25, no. 1, pp. 32-51, 2015.

[22] K. Lu, Y. Xia, C. Yu, and H. Liu, "Finite-Time Tracking Control of Rigid Spacecraft Under Actuator Saturations and Faults," IEEE Transactions on Automation Science and Engineering, vol. 13, no. 1, pp. 368-391, 2016.

[23] C.-W. Chang, C.-F. Hsu, and T.-T. Lee, "Backstepping-Based Finite-Time Adaptive FUZzy Control of Unknown Nonlinear Systems," International Journal of Fuzzy Systems, vol. 20, no. 8, pp. 2545-2555, 2018.
[24] C. Yang, Y. Jiang, W. He, J. Na, Z. Li, and B. Xu, "Adaptive Parameter Estimation and Control Design for Robot Manipulators with Finite-Time Convergence," IEEE Transactions on Industrial Electronics, vol. 65, no. 10, pp. 8112-8123, 2018.

[25] X. Liu, G. Gu, and K. Zhou, "Robust stabilization of MIMO nonlinear systems by backstepping," Automatica, vol. 35, no. 5, pp. 987-992, 1999.

[26] J. Zhou and C. Wen, Adaptive backstepping control of uncertain systems: Nonsmooth nonlinearities, interactions or timevariations, Springer, Berlin, Germany, 2008.

[27] B. L. Cong, X. D. Liu, and Z. Chen, "Backstepping based adaptive sliding mode control for spacecraft attitude maneuvers," Aerospace Science and Technology, vol. 30, no. 1, pp. 1-7, 2013.

[28] J. Yu, L. Zhao, H. Yu, C. Lin, and W. Dong, "Fuzzy finitetime command filtered control of nonlinear systems with input saturation," IEEE Transactions on Cybernetics, vol. 48, no. 8, pp. 2378-2387, 2018.

[29] J. Yu, P. Shi, and L. Zhao, "Finite-time command filtered backstepping control for a class of nonlinear systems," Automatica, vol. 92, pp. 173-180, 2018.

[30] K. B. Ngo, R. Mahony, and Z. Jiang, "Integrator backstepping using barrier functions for systems with multiple state constraints," in Proceedings of the 44th IEEE Conference on Decision and Control, and the European Control Conference (CDC-ECC '05), pp. 8306-8312, December 2005.

[31] K. P. Tee, S. S. Ge, and E. H. Tay, "Barrier Lyapunov functions for the control of output-constrained nonlinear systems," Automatica, vol. 45, no. 4, pp. 918-927, 2009.

[32] Y.-J. Liu and S. Tong, "Barrier Lyapunov functions for Nussbaum gain adaptive control of full state constrained nonlinear systems," Automatica, vol. 76, pp. 143-152, 2017.

[33] Q. Wu and Y. Guo, "Neural Back-Stepping Control of Hypersonic Flight Vehicle with Actuator Fault," Journal of Control Science and Engineering, vol. 2018, Article ID 2198423, 5 pages, 2018.

[34] A. Ilchmann, E. P. Ryan, and S. Trenn, "Tracking control: Performance funnels and prescribed transient behaviour," Systems \& Control Letters, vol. 54, no. 7, pp. 655-670, 2005.

[35] S. Wang, X. Ren, J. Na, and T. Zeng, "Extended-state-observerbased funnel control for nonlinear servomechanisms with prescribed tracking performance," IEEE Transactions on Automation Science and Engineering, vol. 14, no. 1, pp. 98-108, 2017.

[36] Q. Chen, X. Tang, Y. Nan, and X. Ren, "Finite-time neural funnel control for motor servo systems with unknown input constraint," Journal of Systems Science \& Complexity, vol. 30, no. 3, pp. 579-594, 2017.

[37] C. P. Bechlioulis and G. A. Rovithakis, "Robust adaptive control of feedback linearizable MIMO nonlinear systems with prescribed performance," IEEE Transactions on Automatic Control, vol. 53, no. 9, pp. 2090-2099, 2008.

[38] M. Chen, Q. Wu, C. Jiang, and B. Jiang, "Guaranteed transient performance based control with input saturation for near space vehicles," Science China Information Sciences, vol. 57, no. 5, pp. $1-12,2014$.

[39] J. Na, Y. Huang, X. Wu, G. Gao, G. Herrmann, and J. Z. Jiang, "Active adaptive estimation and control for vehicle suspensions with prescribed performance," IEEE Transactions on Control Systems Technology, pp. 1-15, 2017.

[40] C. Yang, X. Wang, L. Cheng, and H. Ma, "Neural-learningbased telerobot control with guaranteed performance," IEEE Transactions on Cybernetics, vol. 47, no. 10, pp. 3148-3159, 2017. 
[41] R. Sun, J. Na, and B. Zhu, "Robust approximation-free prescribed performance control for nonlinear systems and its application," International Journal of Systems Science, vol. 49, no. 3, pp. 511-522, 2018.

[42] C. Ming, R. Sun, and B. Zhu, "Nonlinear fault-tolerant control with prescribed performance for air-breathing supersonic missiles," Journal of Spacecraft and Rockets, vol. 54, no. 5, pp. 10921099, 2017.

[43] Q. Hu, X. Shao, and L. Guo, "Adaptive fault-Tolerant attitude tracking control of spacecraft with prescribed performance," IEEE/ASME Transactions on Mechatronics, vol. 23, no. 1, pp. 331341, 2018.

[44] B. Huo, Y. Xia, L. Yin, and M. Fu, "Fuzzy Adaptive FaultTolerant Output Feedback Attitude-Tracking Control of Rigid Spacecraft," IEEE Transactions on Systems, Man, and Cybernetics: Systems, vol. 47, no. 8, pp. 1898-1908, 2017.

[45] J. L. Crassidis and F. L. Markley, "Sliding mode control using modified Rodrigues parameters," Journal of Guidance, Control, and Dynamics, vol. 19, no. 6, pp. 1381-1383, 1996.

[46] A. Levant, "Higher-order sliding modes, differentiation and output-feedback control," International Journal of Control, vol. 76, no. 9-10, pp. 924-941, 2003.

[47] Q. Chen, L. Shi, J. Na, X. Ren, and Y. Nan, "Adaptive echo state network control for a class of pure-feedback systems with input and output constraints," Neurocomputing, vol. 275, pp. 13701382, 2018.

[48] C. Yang, Y. Jiang, Z. Li, W. He, and C.-Y. Su, "Neural control of bimanual robots with guaranteed global stability and motion precision," IEEE Transactions on Industrial Informatics, vol. 13, no. 3, pp. 1162-1171, 2017.

[49] G. H. Hardy, J. E. Littlewood, and G. Pólya, Inequalities, Cambridge University Press, London, UK, 2nd edition, 1952.

[50] S. P. Bhat and D. S. Bernstein, "Finite-time stability of continuous autonomous systems," SIAM Journal on Control and Optimization, vol. 38, no. 3, pp. 751-766, 2000. 


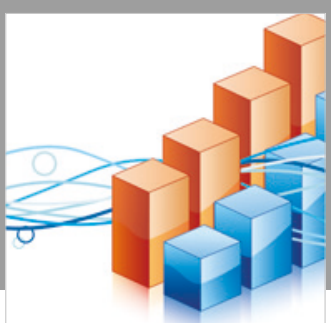

Advances in

Operations Research

\section{-n-m}
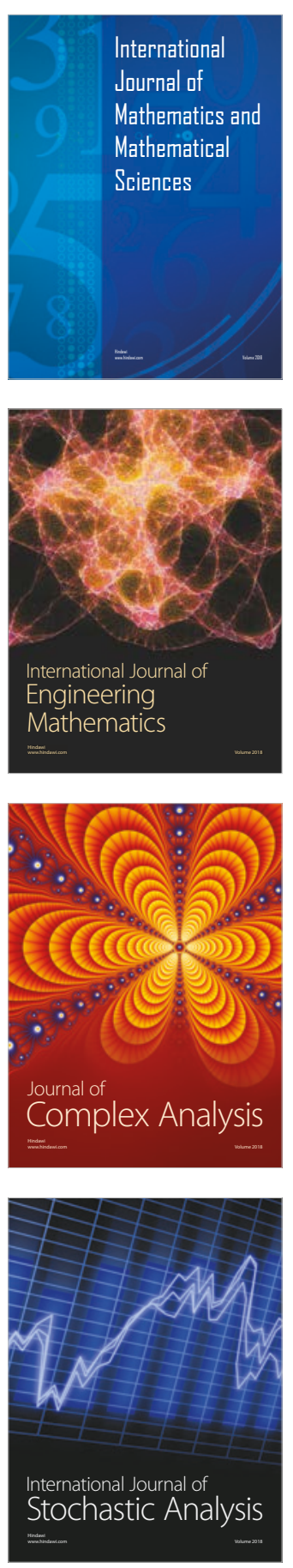
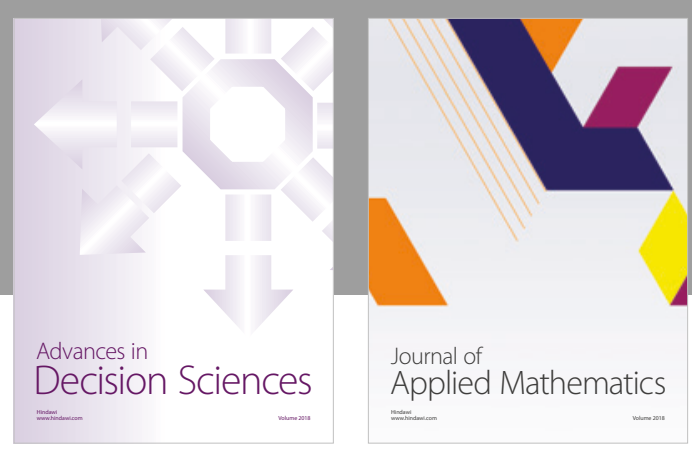

Journal of

Applied Mathematics
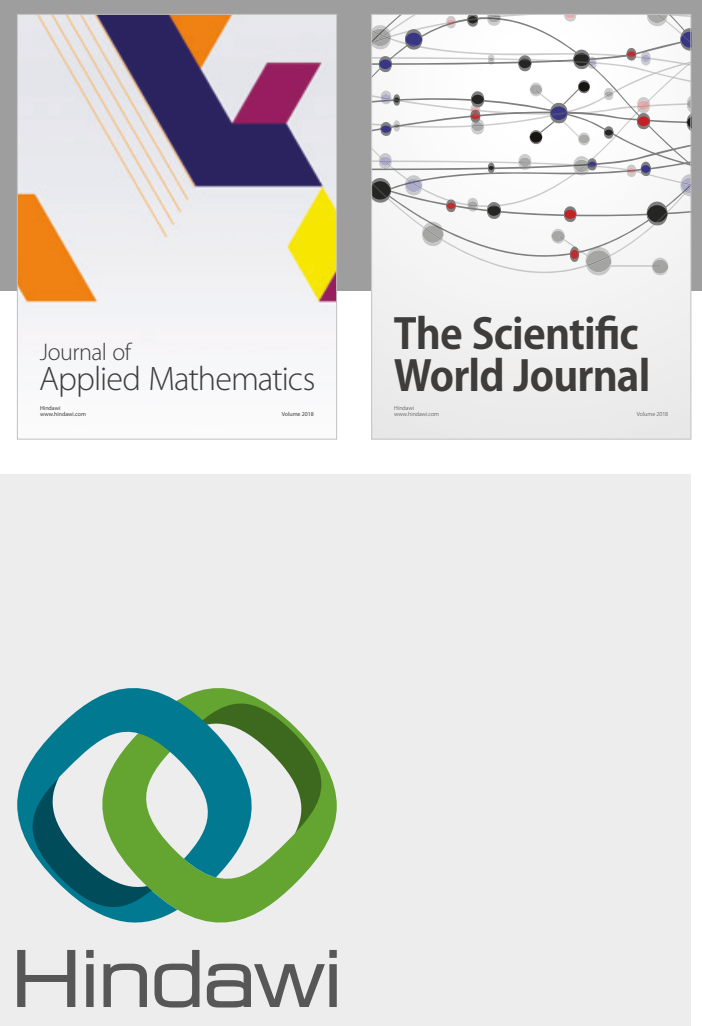

Submit your manuscripts at

www.hindawi.com

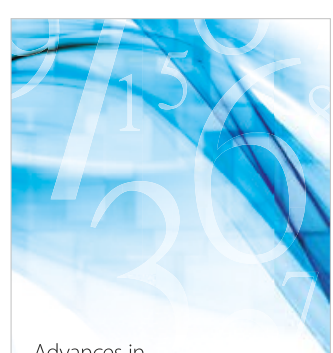

Advances in
Numerical Analysis
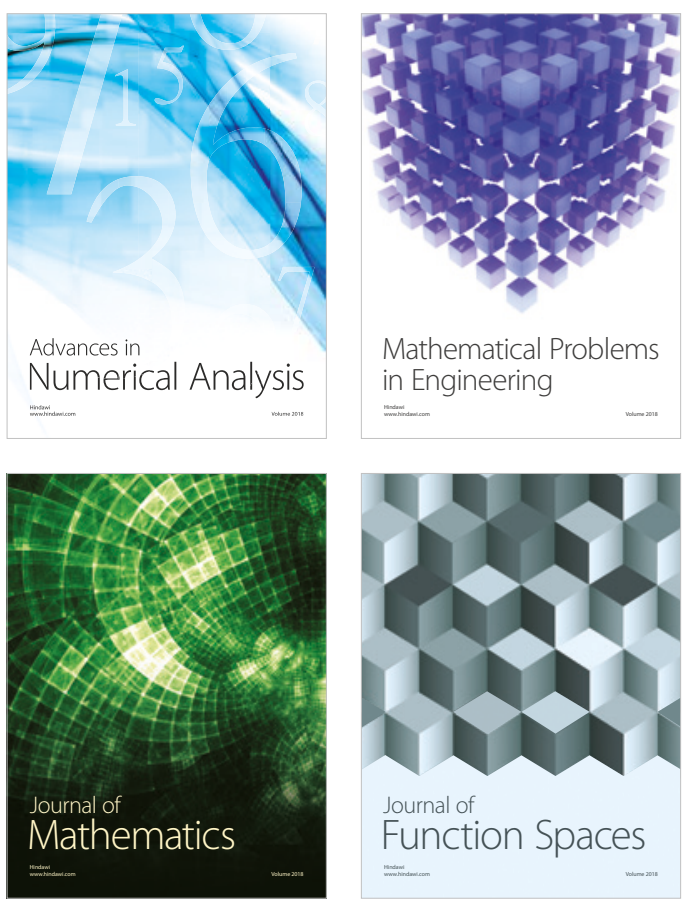

Mathematical Problems in Engineering

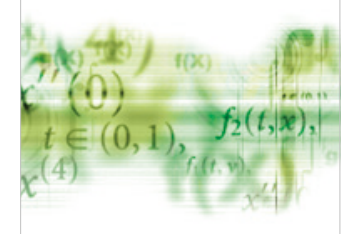

International Journal of

Differential Equations

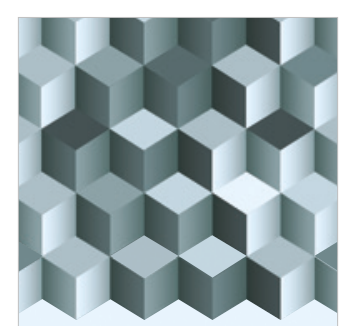

Journal of

Function Spaces
The Scientific

World Journal

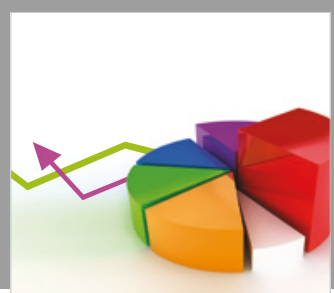

Journal of

Probability and Statistics
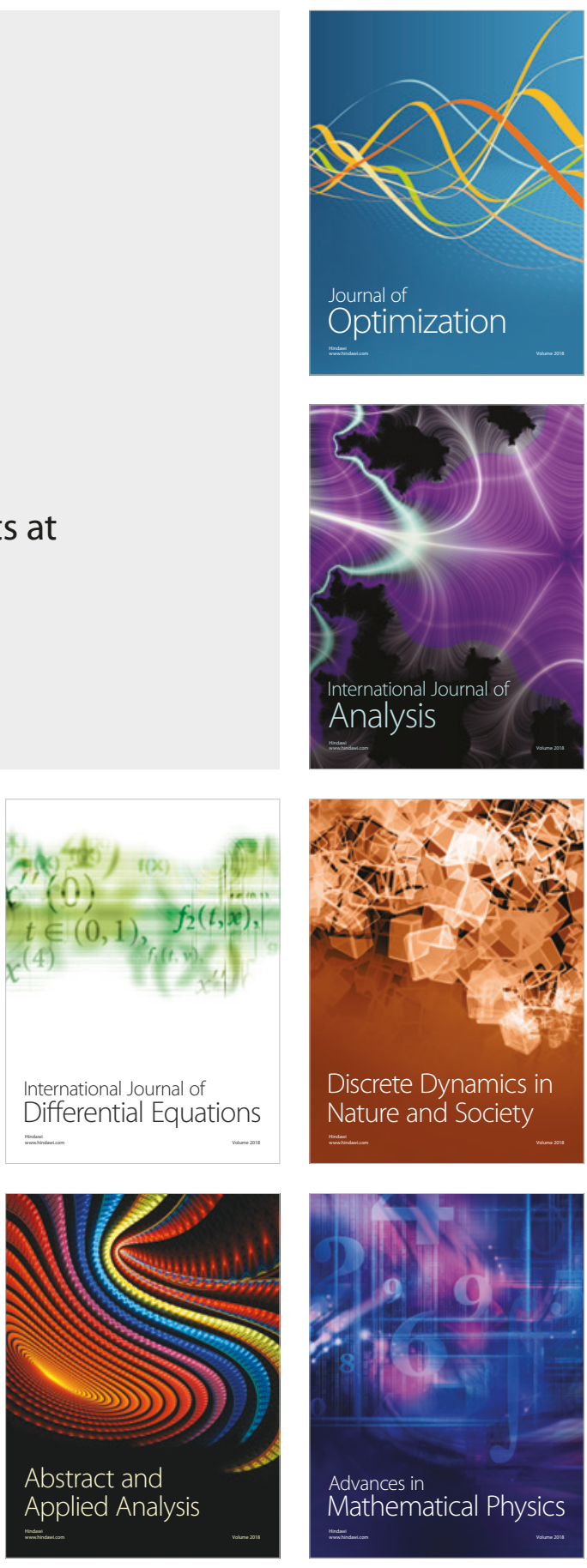